\title{
QoE-aware Video Streaming over Integrated Space and Terrestrial 5G Networks
}

\author{
Satish Kumar, Ning Wang, Yogaratnam Rahulan, Barry Evans
}

\begin{abstract}
The integration of Space Information Network (SIN) with terrestrial infrastructures has been attracting significant attentions in the context of $5 \mathrm{G}$, where satellite communications can be leveraged as additional capabilities such as backhauling between the core network and remote mobile edge sites. However, simple addition of SIN capabilities to terrestrial 5G does not automatically lead to enhanced service performance without systematic scheduling of coexisting resources. In this article we focus on the scenario of multi-link video streaming over both parallel Geostationary Earth Orbit (GEO) satellite and terrestrial 5G backhaul links for enhancing user Quality of Experience (QoE) and network efficiency. The distinct challenge is the complex optimization of scheduling video segment delivery via two parallel channels with very different characteristics while striving to enhance the video quality and resource optimality. We carried out systematic experiments based on a real-life 5G testing framework with integrated GEO satellite and terrestrial backhaul links. The experimental results demonstrate the effectiveness of our proposed 5G edge computing based solution for holistically achieving assured user experiences and optimised network resource efficiency in terms of video traffic offloading.
\end{abstract}

Index Terms-HTTP Adaptive Streaming, Satellite and Terrestrial network integration, 5G networks, Quality of Experiences, Scalable Video Coding

\section{INTRODUCTION}

$\mathbf{T}$ HE key objective of Enhanced Mobile Broadband (eMBB) service is to deliver high-quality video content to end-users with assured quality of experiences (QoE). One distinct challenge here is how to sufficiently handle the huge volume of video content traffic in the $5 \mathrm{G}$ backhaul network. In recent years, efforts have been made towards seamless integration of satellite capabilities in $5 \mathrm{G}$ network infrastructures. However, the role of satellite in the upcoming $5 \mathrm{G}$ is still under study in the 3GPP working group.

Traditionally, the satellite network is mainly used for the delivery of content in an offline manner. How to support video on-demand (VoD) streaming over satellite links in the $5 \mathrm{G}$ network environment has been recently studied based on the popular HTTP Adaptive Streaming (HAS) platform [1] . However, there has been little attempt in the community to support the streaming of VoD video content over the integrated satellite and terrestrial 5G networks. Efficient video delivery over integrated satellite and terrestrial link is challenging mainly due to their distinct characteristics in terms of round trip time (RTT). The EU H2020 SaT5G [2] project has aimed to bring Satcom into $5 \mathrm{G}$ by defining satellite-based backhauling and offloading solutions. This project aims to develop and validate the key $5 \mathrm{G}$ technologies in an attempt to explore Satcom capabilities while mitigating their inherent limitations such as long propagation latency. The work reported in this paper represents one use case scenario investigated within the SaT5G Project, for leveraging satellite communications to support QoE-assured content distribution in a $5 \mathrm{G}$ network.

In this work, we propose a novel Video-segment Scheduling Network Function (VSNF) that aims to deliver enhanced quality of Scalable Video Coding (SVC) video content to the end-users by efficiently utilizing both satellite and terrestrial backhaul resources in $5 \mathrm{G}$. The proposed framework can be flexibly embedded by a mobile network operator (MNO) or a content provider as a virtual network function (VNF) at the Mobile Edge Computing (MEC) side [3]. The proposed VSNF framework operates based on five components: Request Handler (RH), Adaptation Module (AM), Link Selection Module (LSM), Enforcer (EF) and Multiplexing Module (MM), all integrated at the MEC server. RH is responsible for handling all the SVC video segment download requests during active VoD sessions. AM and LSM, the two key modules with segment adaptation and scheduling intelligence, are responsible for determining the specific video quality (in terms of the number of enhanced layers to be requested) and which backhaul channel is scheduled to deliver the requested layer(s) for each video segment. EF is the function that actually performs concurrent downloading of different layers of video segments based on HTTP according to the downloading decisions made by AM and LSM. Finally, MM multiplexes the downloaded SVC layers and sends them to the corresponding client. Detailed specification on the working mechanisms of these components will be presented in Section IV. The rest of the paper is organised as follows. The detailed specification on the satellite and terrestrial integrated 5G system along with the HAS system is provided in Section II. Related work is presented in section III. In section IV, we present the MEC-based VSNF framework and the supporting optimization algorithms for achieving the aforementioned objectives. Reallife experimental details and results are provided in section $\mathrm{V}$, followed by the final conclusions in section VI.

\section{SySTEM OVERVIEW}

\section{A. Satellite and terrestrial Integrated $5 G$ network}

Fig. 1 depicts an overview of a Satellite and Terrestrial integrated $5 \mathrm{G}$ network system based on 3GPP architecture. The 5G infrastructure broadly comprises of two components, namely core network and mobile edge network. In satellite and terrestrial integrated $5 \mathrm{G}$ system, the mobile edge network is connected to the central $5 \mathrm{G}$ core network via two backhaul links, namely terrestrial link and satellite link. The satellite backhaul link is enabled by installing a Non-Terrestrial Network (NTN as defined in 3GPP) at the mobile edge and 


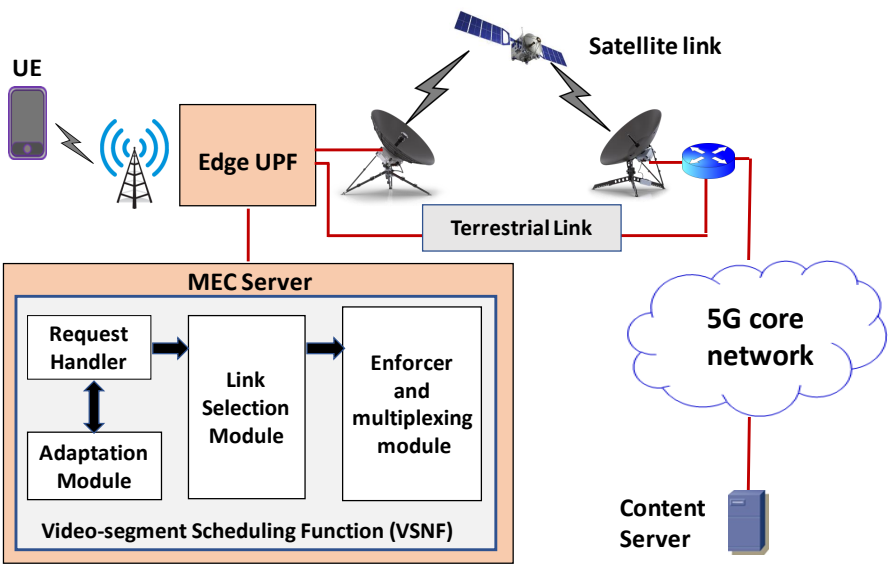

Fig. 1. System Overview of Satellite and Terrestrial integrated 5G Network

deploying an NTN gateway at the $5 \mathrm{G}$ core side. The proposed $V S N F$ is deployed at the MEC server as a VNF.

\section{B. SVC based HTTP Adaptive Streaming}

In an attempt to deliver enhanced video viewing experience to all clients, HAS [1] has emerged as a de-facto technology. $H A S$ optimizes the overall transmission quality of a service provider by dynamically adapting video transmission bit-rates over time, based on mobile device capability and estimated network conditions. In $H A S$, the server encodes the video content at various bit-rates and permits dynamic bit rate adaptations of a flow. In this work, we have used SVC as an encoding technology. In SVC, video content is encoded into one base layer plus multiple enhancement layers on per segment basis, allowing on-the-fly bit-rate adaptation according to available bandwidth resources during a video session.

\section{RELATED WORK}

HAS has emerged as a de facto technology that enables a service provider to deliver enhanced video viewing experience to end users [1]. Authors in [4] have conducted an experimental evaluation of rate adaptation algorithms and concluded that the adaptive HTTP players are still in their infancy and efficient design of adaptive strategies that work on top of complex transport protocol (TCP) is still yet to be investigated. Authors in [5] designed a purely client-side buffer-based adaptation strategy to optimise QoE. They concluded that playback freezing and bit-rate switching can be avoided by maintaining the playback buffer above a certain threshold. Nguyen et. al. have evaluated the effects of segment duration to video streaming performance under various mobile network conditions [6]. With experimental analysis they reported that the choice of segment duration depends on various factors such as end-to-end delay, server and network infrastructure, and client-side buffer length. Authors in [7] presented a 5Goriented network architecture that is based on a satellite link as a backhaul and MEC to support live eMBB application. Araniti et. al. have presented a combination of use of multicast and resource allocation strategy based on subgrouping and application layer joint coding to enhance the performance of live video streaming in mobile satellite systems [8].

Multiple research initiatives has been working towards efficient integration of Space Information Network (SIN) in 5G. Shared Access Terrestrial-Satellite Backhaul Network enabled by Smart Antennas [9] is a Horizon 2020 to boost the mobile backhaul performance in terms of resilience and capacity while assuring efficient spectrum use. Furthermore, the EU H2020 project, Virtualized Hybrid Satellite-Terrestrial Systems for Resilient and Flexible Future Networks [10] is another project addressing the integration of satellite and terrestrial networks and attempts to utilize Software-defined networking and network function visualization in novel ways.

\section{Proposed Strategy}

In this work, we propose a Video-segment Scheduling Network Function (VSNF) for satellite and terrestrial integrated $5 \mathrm{G}$ network environments, aiming to achieve both enhanced user QoE and optimized resource utilizations in dynamic conditions. As depicted in Fig. 1, the proposed strategy is deployed at the MEC server. The proposed framework is designed as a modularized architecture, which helps to efficiently utilise both the backhaul links along with MEC functionalities. The VSNF operates using five components namely, Request Handler (RH), Adaptation Module (AM), Link Selection Module (LSM), Enforcer (EF), and multiplexing Module (MM). All the components cooperatively work in together to deliver satisfactory QoE to each active UE.

From the UE's perspective, VSNF is responsible for handling all the video segment download requests during the $\operatorname{VoD}$ session. Each UE sends segment download requests to the $R H$. The $A M$ periodically decides to download a specific number of segments (through intelligent prefetching techniques ahead of actual requests from UE) corresponding to each UE at a particular enhancement layer based on the estimated backhauled link condition and aggregate bit-rate demands. In this work, we assume that each UE has sufficient access network radio resources and hence the focus is on the backhaul conditions of the parallel satellite and terrestrial links. The download request for all the layers selected by the adaptation module is forwarded to $L S M$ which chooses a backhaul link (satellite or terrestrial) for downloading each segment layer. Based on the decisions made by LSM, $E F$ downloads the video segment via the selected link. Finally, when all the enhancement layers corresponding to a particular segment have downloaded, the $M M$ merges all the layers (SVC format) and generates an $\mathrm{H} 264$ file (AVC format). The next subsection provides a detailed description of all the components of VSNF.

\section{A. Request Handler}

The Request Handler (RH) handles all the segment download requests from the UEs. The requested segment is served immediately to the respective UE if it is available at the MEC server. Otherwise, the $R H$ waits until the layers corresponding to the requested segment have been successfully downloaded. 


\section{B. Adaptation Module}

At each adaptation interval boundary (typically in the range of 5 to $10 \mathrm{secs}$ ), the Adaptation Module (AM) dynamically adapts the enhancement layer on per segment basis for each ongoing video flow. The primary objectives of the $A M$ during the enhancement layer selection are: (i) The overall bit-rate demands should not surpass the estimated backhauled capacity, and (ii) Guaranteed QoE is delivered to each UE. Among the major factors that influence user QoE, the most important ones can be considered in the following contexts.

1) Stable and sustainable buffer conditions: Stalling-free video can be delivered to all end-users by continuously maintaining the playback buffer above a certain threshold. To achieve a stable buffer, $A M$ dynamically decides the Buffer filling ratio for each user based on its instantaneous playback buffer against the targeted buffer threshold. In this work, the buffer filling ratio is an exponential function of difference between targeted buffer and playback buffer. The buffer filling ratio is greater than one if the buffer threshold is greater than playback buffer of the client. Therefore, the designed buffer filling rate function automatically tunes the buffer replenishment priority of client and attempts to steer its playback buffer towards the targeted thresholds.

2) Bit-rate switching or Stability Index: A larger variation in video quality drives to higher perceptual impact during switching. Another crucial aspect of switching modeling is the forgiveness effect. It captures the psychological observation that the quality distortion impact degrades over time. In this work, we define the switching index in the ensuing adaptation interval as an exponential moving average of the enhancement layer switched encountered by the flow in the past adaptation interval [11].

3) Video Quality and Utility Value: In $H A S$, the server stores each video content at various enhancement layers associated with distinct bandwidth demand. The adaptation module decides an enhancement layer for each adaptive video flow. The adaptation module first calculates the bit-rate demands at each enhancement layer for each flow which is equal to the multiplication of encoding bit-rate of the layer and buffer filling rate of the flow.

The adaptation module selects enhancement-layer on per segment basis for each flow such that the aggregate utility value is maximized. The utility value is defined as a function of two parameters: (i) Video encoding quality (or bit-rate demand) and (ii) Stability Index. The utility value of a flow at an enhancement layer is calculated by dividing bit-rate demands by stability index. The division with stability index will restrict the bit-rate switching for the flows having a relatively higher switching in the past.

4) Enhancement Layer Selection: This module selects an enhancement layer for each flow at the boundary of a commencing adaptation interval such that the overall utility of the system is maximized while the aggregate bit-rate demand over all flows must not exceed the overall capacity of the satellite and terrestrial backhauled integrated 5G network. We have mapped the enhancement layer selection to the Multiple Choice Knapsack Problem [12]. Here, each flow is analogues to the item, bit-rate demand at each layer is analogues to weight, the utility value is analogues to reward, and satellite and terrestrial integrated backhaul capacity is analogues to knapsack capacity. We have solved the mapped enhancement layer selection problem using Dynamic Programming (DP) algorithm [12] after discretizing the capacity constraint. The computational complexity of DP based solution strategy is $O(N \times L \times C)$. Here, $N$ represents the total number of concurrent flows, $L$ denotes the total number of layers (base and enhancement), and $C$ is the total discrete capacity of satellite and terrestrial integrated $5 \mathrm{G}$ network.

After selecting the enhancement layer for each flow, the adaptation module calculates the number of segment needed to be downloaded (prefetched) in the ensuing adaptation interval based on the buffer filling ratio and segment duration. All the segments scheduled to download in the ensuing adaptation interval are fed as an input to the Link Selection Module.

\section{Link Selection Module}

The scheduled layers to be downloaded by the adaptation module are fed as an input to the Link Selector Module (LSM). LSM endeavors to efficiently selects a backhaul link for each layer primarily based on two factors: (i) Segment playback deadline and (ii) base/enhancement layer segment size. The clients having smaller buffers are prioritized to schedule via the terrestrial link to replenish the buffer quickly. To prioritize all clients, the LSM categorizes each video flow into three distinct states: critical, precaution, and stable as follows:

1) Critical State: A flow is considered here as the playback buffer is minimal, and the probability of stalling is very high. The flow moves from critical to precaution state, once the buffer length is greater than a threshold $T h_{c}$. Authors in [5] have conducted a set of experiments with varying threshold values and concluded that the stalling events are negligible by maintaining buffer length above 10 secs. In this work, we have chosen $T h_{c}$ equal to 10 secs.

2) Precaution State: This is a "buffering" state between the critical state and the stable state (mentioned below) when a flow session is not at most vulnerable status but still requires a higher segment-serving priority than those with sufficient video buffer. The threshold between precaution and stable state is $T h_{s}$. A typical threshold value above which the flow is considered to be in stable state is 30 secs [4].

3) Stable State: When the playback buffer of a flow is greater than or equal to $T h_{s}$, the video session is considered to be in the stable state. The probability of stalling is comparatively low in this state.

LSM classifies each of the concurrent flows into one of the states according to its instantaneous buffer length. For the LSM at the MEC server side to have the up-to-date knowledge about the real-time UE buffer conditions, we use our previous work for real-time estimation of the buffer conditions [13].

The second factor considered by the LSM during link selection is segment/layer size. Achieving a high data rate using TCP over satellite is difficult due to its larger RTT [7]. Additionally, the achieved throughput is even lower for the download of smaller file sizes. To quantify this, we have conducted a set of experiments of layers download with 


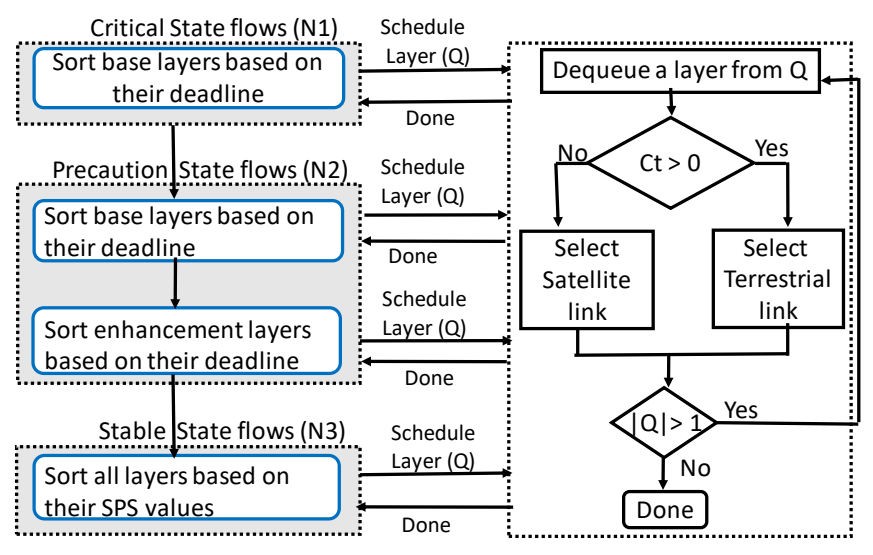

Fig. 2. Flow chart for Link selection Strategy

varying file sizes via a real GEO satellite link. The detailed description of the experimental setup is given in section $\mathrm{V}$. It has been observed from the experiments that the achieved download throughputs via the satellite link are respectively around $2 \mathrm{Mbps}, 4 \mathrm{Mbps}$ and $6 \mathrm{Mbps}$ for files with sizes 2 $\mathrm{MB}, 8 \mathrm{MB}$ and $12 \mathrm{MB}$. As expected, it may be noted that the satellite downloading throughput is higher for the larger file sizes. Therefore, it is desirable to schedule the layers with larger file sizes and comparatively higher deadline via the satellite link. To capture this, we have defined a parameter for each scheduled video segment called Scheduling Priority for Satellite $(S P S)$ which is directly proportional to the segment's file size and inversely proportional to the segment's playing deadline.

A flow chart diagram for link selection strategy is presented in Fig. 2 which works in three rounds. In the first round, the link for critical state flows is selected, followed by precaution state flows in the second round and then, stable state flows are in the third round. Let the total number of concurrent flows in critical, precaution and stable state be $N_{1}, N 2$, and $N_{3}$, respectively.

In the first round, the base layer of the critical state flows is sorted based on their playing deadline and stored into a queue $Q$. Then, Schedule Layer $(Q)$ selects a link for each in $Q$ through the terrestrial link if its bandwidth demand is less than the available bandwidth of the terrestrial link. Otherwise, the satellite link is selected for the base layer. The time complexity for sorting the layers and scheduling them over the backhaul link is $O\left(N_{1} \log N_{1}\right)+O\left(N_{1}\right)$, which is equals to $O\left(N_{1} \log N_{1}\right)$.

After selecting the link for critical state layers, layers belonging to the precaution state are scheduled in the second round. The backhaul link selection for precaution state flows is performed based on two parameters: (i) Playing deadline of the layers and (ii) Layer's priority in the multiplexing. The link selection strategy attempts to select the terrestrial link for the base layer or layers having a lower playing deadline. To achieve this, firstly, the base layers of the precaution state flows are sorted based on their playing deadline and scheduled through the Schedule Layer $(Q)$ routine (time complexity is $O\left(N_{2} \log N_{2}\right)$ ). Then, link selection for enhancement layers of precaution state flows is performed. The time complexity for sorting the layers and scheduling them over backhaul link is
$O\left(\left(N_{2} \times(L-1)\right) \log \left(N_{2} \times(L-1)\right)\right.$, where $\mathrm{L}$ is the total number of layers (base + enhancement).

In the third round, the strategy selects a link for stable state flows. It may be noted that the probability of stalling for stable state flows is very low. Therefore in this round, the objective is to schedule layers with larger file sizes via the satellite link. This helps the system to achieve higher overall throughput via the satellite backhaul link. The link selection strategy first sorts all the layers of stable state based on their decreasing values of $S P S$ and stores them into queue $Q$. Then, the Schedule $\operatorname{Layer}(Q)$ selects a backhaul link for all the layers in $Q$. The time complexity for sorting the layers and scheduling them over the backhaul link is $O\left(\left(N_{3} \times L\right) \log \left(N_{3} \times L\right)\right.$, where $\mathrm{L}$ is the total number of layers. Therefore, the worst-case time complexity of $L S M$ is $O(N \times \operatorname{Llog}(N \times L))$, where $N$ is the maximum no of flows in any state.

\section{Enforcer and Multiplexing Modules}

Once the link for all the layers is selected by LSM, the Enforcer concurrently establishes the HTTP connections from the MEC to the content server via the selected backhaul link.

The Multiplexing module merges all the downloaded layers (in SVC file format) and creates an AVC file (H264 file format). In this work, we have offloaded the multiplexing procedure from the player to the MEC server and attempted to reduce the computational load at the player. This is an optional feature that is embedded at the MEC side for realtime transcoding from SVC to AVC to reduce the decoding complexity of SVC video for UE with limited processing capabilities. In the case a UE is able to directly decode SVC video segments, then MM is not required. The Multiplexing procedure is triggered in two scenarios: (i) Once all the layers corresponding to a requested segment are downloaded and (ii) As the playing deadline of a client approaches and the base layer corresponding to the requested segment is downloaded at the MEC server. This provides flexibility to the framework in delivering at least base quality to end-users and avoids any buffering event due to buffer shortage. Finally, the generated AVC file is forwarded by the Request Handler to the corresponding UE.

\section{EXPERIMENTS AND RESULTS}

\section{A. Experiment Setup}

The proposed VSNF framework has been implemented on a real Satellite and Terrestrial integrated 5G (SaT5G) network [2]. The experimental environment is set up as follows:

1) SaT5G Network: In this work, we have considered a multi-client scenario in which six UEs are requesting SVC video from the content server using the SaT5G network. Fig. 3 shows an overview of the network topology used for the experiment. The SaT5G project created a satellite backhaul link via a satellite Vsat terminal along with modem installed on the roof of the 5G Innovation Centre (University of Surrey) Building and a Satellite Gateway installed at Goonhilly in the UK. A two-way connection was established between satellite terminal and satellite gateway via Avanti's Hylas-4 GEO Satellite. The satellite gateway includes virtualized core and 


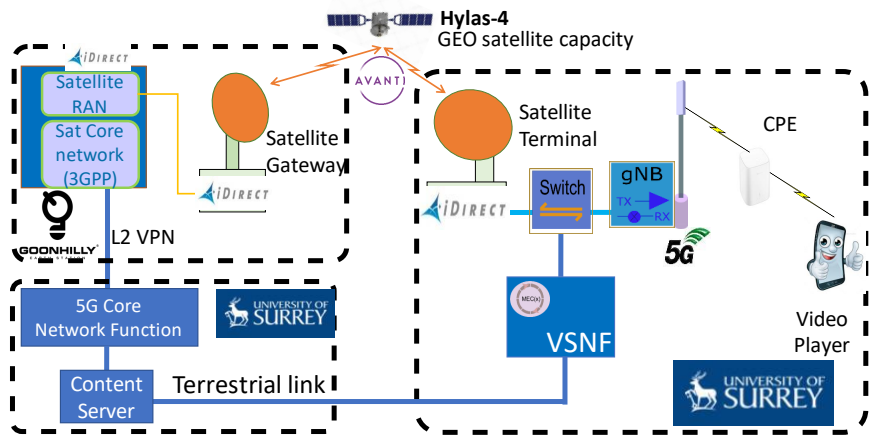

Fig. 3. Network Topology Setup for Content Delivery over Satellite and Terrestrial integrated 5G Network

RAN components enabling it to appear as a $5 \mathrm{G}$ gNB. The satellite gateway is connected to the $5 \mathrm{G}$ core (available at the 5G Innovation Centre, University of Surrey, UK) via a L2 VPN connection. The SVC video server is connected to the $5 \mathrm{G}$ core network side. For proof-of-concept purpose, a low capacity backhaul terrestrial link, we have created an emulated link with $50 \mathrm{Mbps}$ capacity between the MEC side and the content server, whose delay is tunable, representing different distances between the two sites.

2) SVC Video Content and media player: We used SVC toolchain for creating SVC layers along with video segment and playing it at the client-side [14], [15]. We have stored video stream Big Bug Bunny (BBB) at base layer (BL) and three enhancement layers (EL) at the content server. The encoding bit-rate of $B B B$ at $B L, E L_{1}, E L_{2}$, and $E L_{3}$ are 4.02 Mbps, 5.6 Mbps, 7.15 Mbps and 11.01 Mbps, respectively. Further, the $B B B$ video stream is available at three distinct segment duration (SD), namely 2 secs, 4 secs, and 8 secs.

\section{B. Results}

To benchmark the performance of the proposed scheme, we have setup three network scenarios (represented as S1, S2, S3).

1) Scenario S1: Only a terrestrial backhaul link (with capacity $50 \mathrm{Mbps}$ and latency $10 \mathrm{~ms}$ ) is available. Adaptation algorithm, which is available at the client-side, selects an enhancement layer for each segment based on the estimated network condition. All request goes to the MEC server via the $\mathrm{gNB}$, and the MEC server chooses terrestrial backhaul link to download all the layers.

2) Scenario S2: In this scenario, both terrestrial (with capacity $50 \mathrm{Mbps}$ and latency $10 \mathrm{~ms}$ ) and satellite backhaul links are available. The Adaptation algorithm (same as in scenario S1), which is available at the client-side, selects an enhancement layer for each segment based on the estimated network condition. The layer download requests from all the clients go to the MEC server via the gNB. The MEC server selects a backhaul link for each layer using first-come-firstserve scheduling without taking into account any context information on the UE buffering status. In this scheduling strategy, the layer download requests from all the clients are first enqueued into a common queue, and the scheduler first tries to schedule all the layers via the terrestrial backhaul

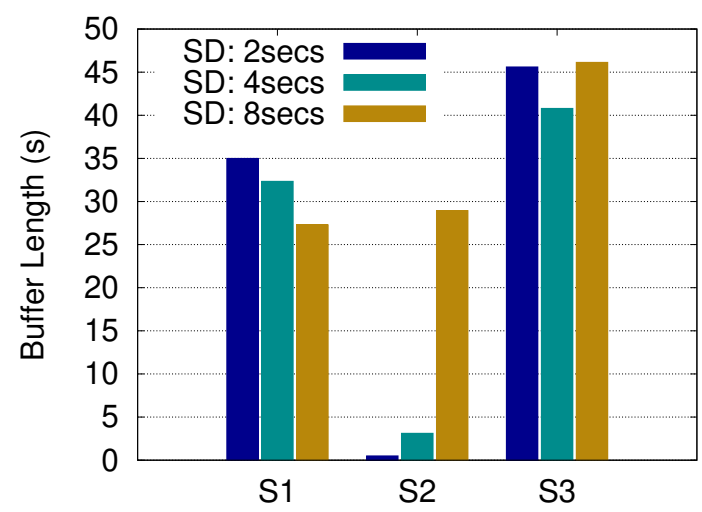

Fig. 4. Average Playback Buffer achieved by the client

link. The satellite backhaul link is selected to download layers once the terrestrial backhaul link becomes overloaded. It can be inferred that, such a context-agnostic scheme may possibly lead to even worse user QoE than in S1. This is because if a video segment (in particular the base layer) is blindly scheduled on to the satellite link with long latency, the corresponding downloading time will substantially increased, resulting in buffer starving and also affecting the adaptation decision on subsequent segment requests.

3) Scenario S3: In scenario S3, the proposed scheme with Satellite and terrestrial (with capacity $50 \mathrm{Mbps}$ and latency $10 \mathrm{~ms}$ ) integrated 5G network are available. The proposed framework $V S N F$ is deployed at the MEC server. In this scenario, each client/UE sends segment download requests to the MEC server, and the proposed VSNF (available at MEC server) handles all the requests during the entire video session.

In scenarios $S 1$ and $S 2$, the default client-side bit-rate adaptation strategy applied as benchmark scheme to select a layer for each segment of the video session [14], [15]. On the other hand, the adaptation module of the proposed framework is used for in-network bit-rate adaptation strategy in scenario S3. Therefore, with the help of these scenarios, we have attempted to compare the client-side bit-rate adaptation strategy (without any intelligence available inside the network) with our proposed framework. In each network scenario $(S 1, S 2$, or $S 3$ ), the performance of multi-client scenarios has been measured in which six distinct clients are requesting SVC videos via the SaT5G network using VoD. Further, each set of experiments has been carried out with three distinct sets of segment duration (SD), namely 2 secs, 4 secs, and 8 secs.

Fig. 4 depicts the average playback buffer achieved by each client in all the considered scenarios (S1, S2, and S3) with segment length equal to 2 secs, 4 secs, and 8 secs. It may be noted from the figure that the playback buffer achieved by the client is typically lower during scenario $S 2$ as compared to $S 1$ and $S 3$. Further, the client has lower buffer size during $S 1$ as compared to $S 3$. The lower playback buffer during $S 1$ as compared to $S 3$ is mainly because of limited terrestrial bandwidth, which is shared among all the clients. Further, it may be noted from Fig 4 that the playback buffer typically decreases after integrating the satellite link in addition to the terrestrial backhaul link in scenario $S 2$. In 


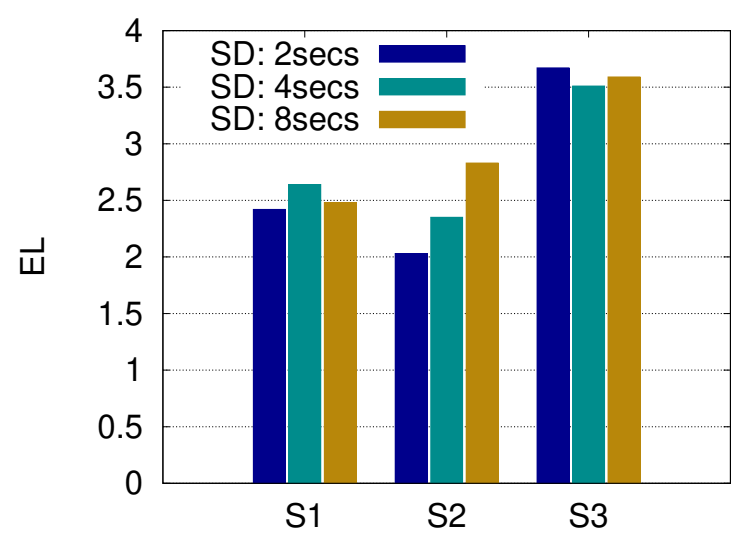

Fig. 5. Average Enhancement Layer downloaded by each client

$S 2$, the segment/layer download request from all the clients are first organized into a First In First Out (FIFO) queue at the MEC server. Then, the schedule first attempts to schedule each layer in the queue via the terrestrial link if the terrestrial backhaul has enough remaining capacity. Otherwise, the layers are scheduled via the satellite link. This naive scheduling may cause longer segment downloading time, potentially leading towards buffer starvation. However, with the increasing value of segment duration, the playback buffer increases, as may be observed from Fig. 4. This may be attributed to the fact that the achieved downloading throughput for the layers having larger file size is comparatively higher via the satellite link.

The low downloading throughput via satellite backhaul is mainly due to three reasons: (i) All the files are downloaded using HTTP over TCP protocol, (ii) The latency of the GEO satellite link is large (around $500 \mathrm{~ms}$ ) and (iii) The file sizes of most of the layers are very small for the smaller video segment duration. Therefore, the clients may experience very low downloading throughput when their layers are scheduled via the satellite link, leading to higher buffering events during scenario S2. However, the performance of clients significantly improves during scenario $S 2$ with larger segment duration videos. On the other hand, it may be observed from Fig 4 that the proposed framework (VSNF) is able to maintain high playback buffer in all scenarios. Maintenance of stable playback buffer is achieved by the proposed strategy mainly by a combination of three mechanisms: (i) Buffer filling ratio adjustment based on the instantaneous playback buffer, (ii) classification of clients in the three states (critical, precaution, and stable) during link selection module and (iii) the proposed enhancement layer adaptation module which is aware of instantaneous SaT5G link capacity as well as traffic load.

Fig. 5 depicts the average enhancement layer downloaded by the clients. It may be observed from the figure that the proposed strategy (Scenario S3) is able to deliver video with higher quality to the clients as compared to the other two scenarios $\left(S_{1}\right.$ and $\left.S_{2}\right)$. The better performance achieved by the proposed framework is mainly: (i) Optimally utilizing both the backhaul links during layer selection by the adaptation module and link selection module, (ii) Carefully prefetching the scheduled segments for all clients for the ensuing

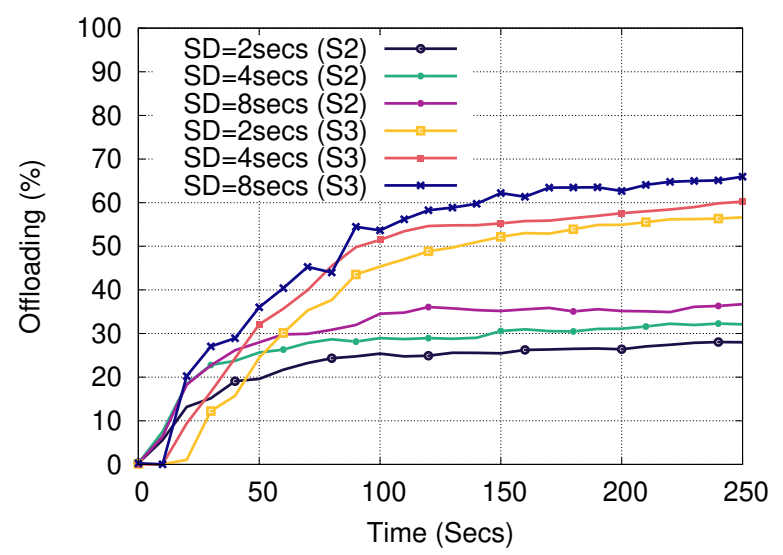

Fig. 6. Offloading (\%) for scenarios $S 2$ and $S 3$ with SD 2, 4, 8 secs

adaptation duration by adaptation module and link selection module and (iii) Concurrently establishing multiple HTTP connections to download all layers via the selected backhaul link by the enforcer module. The prefetching, caching, and multiple concurrent layers download via both the backhaul links help the proposed framework to efficiently utilize the available high capacity and long latency satellite link.

Fig. 6 depicts the achieved offloading percentage of traffic from terrestrial to satellite backhaul link in two scenarios $S 2$ and $S 3$ while clients are requesting video at three segment durations ( $\mathrm{SD}=2 \mathrm{secs}, \mathrm{SD}=4 \mathrm{secs}$, and $\mathrm{SD}=8 \mathrm{secs}$ ). It may be observed from the figure that the proposed strategy is able to offload more than $50 \%$ of the traffic to the satellite backhaul link in almost all scenarios and, thus, be able to utilize both the backhaul links. On the other hand, approximately $30 \%$ of the video traffic has been downloaded via the satellite link during scenario $S 2$. The improved offloading percentage achieved by the proposed framework is primarily due to the following reasons: (i) Classification of flows into different classes and schedule layer download via both links by the link selection module, (ii) Prefetching multiple segments at the MEC and scheduling non-urgent layers via the satellite link by the adaptation module and link selection module, respectively, and (iii) Prioritizing download of layers with larger segment duration (larger file size) via the satellite link by the link selection module which ultimately gives higher download throughput. Additionally, it may be observed from the figure that the achieved offloading percentage is higher with the longer segment duration due to the improved performance of the satellite link with the larger file sizes.

It may be observed from the above results that the proposed strategy is able to achieve high-quality and stable buffer to end-users with both smaller as well as larger segment duration. However, when the segment duration is 2 secs, the QoE performance of the proposed strategy is slightly better, and the offloading percentage is slightly lower. Therefore, a smaller segment duration may be a good choice for the proposed framework to deliver good QoE, albeit compromised somewhat with the satellite backhaul utilisation. 


\section{CONCLUSION}

In this work, a novel SVC based content delivery framework for a satellite and terrestrial integrated $5 \mathrm{G}$ network has been introduced. The proposed framework is aims at delivering enhanced quality of video viewing experience to the endusers by efficiently utilising both of the available backhaul links. The proposed framework is deployed at the MEC server and utilises prefatching and processing mechanism during the content distribution process. The framework is validated using a real over the air satellite and terrestrial integrated $5 \mathrm{G}$ network. Experimental results reveal that the proposed content delivery framework is able to deliver high quality stalling-free video viewing experience to all the active users by achieving a high degree of content offloading from the terrestrial link to the satellite backhaul link thus saving on limited terrestrial network resources.

\section{ACKNOWLEDGMENT}

This work is funded by EU H2020 SaT5G project under grant number 761413. The authors would also like to acknowledge the support of the University of Surreys 5G/6G Innovation Centre (5GIC/6GIC) (http://www.surrey.ac.uk/5gic) members for this work.

\section{REFERENCES}

[1] M. Seufert, S. Egger, M. Slanina, T. Zinner, T. Hoßfeld, and P. TranGia, "A Survey on Quality of Experience of HTTP Adaptive Streaming," IEEE Communications Surveys Tutorials, vol. 17, no. 1, pp. 469-492, Firstquarter 2015.

[2] B. Evans, N. Wang, Y. Rahulan, S. Kumar, J. Cahill, M. Kavanagh, S. Watts, D.-K. Chau, Y. Begassat, A.-P. Brunel et al., "An integrated satellite-terrestrial 5G network and its use to demonstrate 5G use cases," International Journal of Satellite Communications and Networking, 2021.

[3] "MEC in 5G networks," European Telecommunications Standards Institute, White Paper, June, 2018.

[4] S. Akhshabi, A. C. Begen, and C. Dovrolis, "An experimental evaluation of rate-adaptation algorithms in adaptive streaming over HTTP," in Proceedings of the second annual ACM conference on Multimedia systems, 2011, pp. 157-168.

[5] W. Huang, Y. Zhou, X. Xie, D. Wu, M. Chen, and E. Ngai, "Buffer state is enough: Simplifying the design of QoE-aware HTTP adaptive video streaming," IEEE Transactions on Broadcasting, vol. 64, no. 2, pp. 590-601, 2018.

[6] D. M. Nguyen, L. B. Tran, H. T. Le, N. P. Ngoc, and T. C. Thang, "An evaluation of segment duration effects in HTTP adaptive streaming over mobile networks," in 2015 2nd National Foundation for Science and Technology Development Conference on Information and Computer Science (NICS). IEEE, 2015, pp. 248-253.

[7] C. Ge, N. Wang, I. Selinis, J. Cahill, M. Kavanagh, K. Liolis, C. Politis, J. Nunes, B. Evans, Y. Rahulan, N. Nouvel, M. Boutin, J. Desmauts, F. Arnal, S. Watts, and G. Poziopoulou, "QoE-Assured Live Streaming via Satellite Backhaul in 5G Networks," IEEE Transactions on Broadcasting, vol. 65, no. 2, pp. 381-391, 2019.

[8] G. Araniti, I. Bisio, M. De Sanctis, F. Rinaldi, and A. Sciarrone, "Joint Coding and Multicast Subgrouping Over Satellite-eMBMS Networks," IEEE Journal on Selected Areas in Communications, vol. 36, no. 5, pp. 1004-1016, 2018.

[9] G. Ziaragkas, G. Poziopoulou, J. Núñez-Martínez, J. Baranda, I. Moreno, C. Tsinos, S. Maleki, S. K. Sharma, M. Alodeh, and S. Chatzinotas, "Sansa-hybrid terrestrial-satellite backhaul network: scenarios, use cases, kpis, architecture, network and physical layer techniques," International Journal of Satellite Communications and Networking, vol. 35, no. 5, pp. 379-405, 2017.

[10] V. Consortium et al., "Vital (virtualized hybrid satellite-terrestrial systems for resilient and flexible future networks)," 2017.
[11] S. Kumar, A. Sarkar, and A. Sur, "A resource allocation framework for adaptive video streaming over lte," Journal of Network and Computer Applications, vol. 97, pp. 126 - 139, 2017.

[12] M. Dyer, W. Riha, and J. Walker, "A hybrid dynamic programming/branch-and-bound algorithm for the multiple-choice knapsack problem," Journal of Computational and Applied Mathematics, vol. 58, no. 1, pp. 43-54, 1995.

[13] C. Ge and N. Wang, "Real-time qoe estimation of dash-based mobile video applications through edge computing," in IEEE INFOCOM 2018 IEEE Conference on Computer Communications Workshops (INFOCOM WKSHPS), 2018, pp. 766-771.

[14] C. Kreuzberger, D. Posch, and H. Hellwagner, "A Scalable Video Coding Dataset and Toolchain for Dynamic Adaptive Streaming over HTTP," in Proceedings of the 6th ACM Multimedia Systems Conference, ser. MMSys '15, T. O. Wei, Ed. New York, NY, USA: ACM, mar 2015, pp. 213-218.

[15] J. Liu, "RTP Sender and Recevier for Multicast in MST Mode for SVC," https://github.com/reidliujun/svc/tree/master/client/svc_dash, 2014.

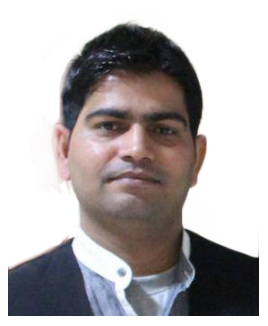

Satish Kumar received his $\mathrm{PhD}$ degrees from the Department of Computer Science and Engineering, Indian Institute of Technology Guwahati, in 2018. Since then, he has been working with 5G/6G Innovation Centre (5GIC/6GIC) at University of Surrey as a Research Fellow. His research interest includes 5G, NFV Management and Orchestration, Multimedia Communication.

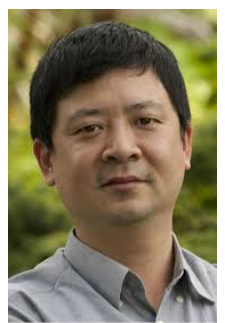

Ning Wang (SM'17) obtained his PhD degree from the Centre for Communication Systems Research (CCSR), University of Surrey in 2004. He is currently a professor at the $5 \mathrm{G} / 6 \mathrm{G}$ Innovation Centre (5GIC/6GIC) at University of Surrey. His research interests include mobile content delivery, 5G networking, edge computing, QoS/QoE and network management.

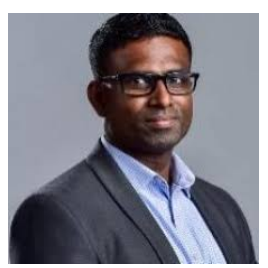

Yogaratnam Rahulan is a Chief Architect at the 5G Innovation Centre, Institute of communications Systems, (ICS), University of Surrey. Rahulan career spans the cellular $3 \mathrm{G}$ and $4 \mathrm{G}$ where he has worked in $\mathrm{R} \& \mathrm{D}$ at Nokia, Accenture, Telefónica $\mathrm{O} 2$ and PPF. Rahulan graduated MSc, BEng (Hons) from University of Surrey, UK.

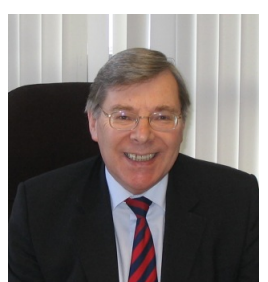

Barry Even Professor Evans has BSC and PhD degrees from the University of Leeds, is a Fellow of the UK Royal Academy of Engineering and of the IET and senior member of IEEE and AIAA. He now heads up satellite communications research in the Institute for Communications Research and 56GIC at Surrey. 